\title{
INFINITE GEODESICS AND ISOMETRIC EMBEDDINGS IN CARNOT GROUPS OF STEP 2
}

\author{
EERO HAKAVUORI
}

\begin{abstract}
In the setting of step 2 sub-Finsler Carnot groups with strictly convex norms, we prove that all infinite geodesics are lines. It follows that for any other homogeneous distance, all geodesics are lines exactly when the induced norm on the horizontal space is strictly convex. As a further consequence, we show that all isometric embeddings between such homogeneous groups are affine. The core of the proof is an asymptotic study of the extremals given by the Pontryagin Maximum Principle.
\end{abstract}

\section{Contents}

1. Introduction

1.1. Structure of the paper

2. Preliminaries

2.1. Stratified groups and homogeneous distances

2.2. The projection norm

2.3. Length structures and sub-Finsler Carnot groups

2.4. Geodesics and blowdowns

2.5. Subdifferentials

3. Step 2 sub-Finsler Pontryagin Maximum Principle

3.1. General statement of the PMP

3.2. The PMP in left-trivialized coordinates

4. Asymptotic behavior of controls

5. Affinity of infinite geodesics

5.1. Sub-Finsler Carnot groups

5.2. Arbitrary homogeneous distances

6. Affinity of isometric embeddings

Acknowledgements

References

Date: November 19, 2019.

2010 Mathematics Subject Classification. 30L05, 53C17, 49K21, $22 \mathrm{E} 25$.

Key words and phrases. Carnot groups, isometries, isometric embeddings, geodesics, sub-Riemannian geometry, sub-Finsler geometry.

The author has been partially supported by the Vilho, Yrjö and Kalle Väisälä Foundation, by the Academy of Finland (grant 288501 'Geometry of subRiemannian groups'), and by the European Research Council (ERC Starting Grant 713998 GeoMeG 'Geometry of Metric Groups'). 


\section{INTRODUCTION}

Carnot groups have rich algebraic and metric structures, and share many properties with normed spaces. Recently several articles have generalized classical regularity results of isometric embeddings in normed spaces into the setting of Carnot groups. In real normed spaces, there are two simple criteria for an isometric embedding to be affine: surjectivity or strict convexity of the norm on the target. Both regularity criteria have analogues for isometric embeddings of Carnot groups.

Surjective isometric embeddings behave in the Carnot group case similarly as they do in the normed-space case. Namely, isometries between arbitrary (open subsets of) Carnot groups are affine [LDO16], i.e., compositions of left translations and group homomorphisms. For globally defined isometries, there is an even more general result that isometries between connected nilpotent metric Lie groups are affine [KLD17.

For non-surjective isometric embeddings, it was proved in Kis03 that if $G$ is a sub-Riemannian Carnot group of step 2, then all isometric embeddings $\mathbb{R} \hookrightarrow G$, i.e., all infinite geodesics, are affine. This property was coined the geodesic linearity property in [BFS18], and was used as an alternative to the strict convexity criterion as the two conditions are equivalent in normed spaces. More precisely, it was shown in [BFS18] that if $\mathbb{H}^{n}$ is a Heisenberg group with a homogeneous distance satisfying the geodesic linearity property, then all isometric embeddings $\mathbb{R}^{m} \hookrightarrow$ $\mathbb{H}^{n}$ and $\mathbb{H}^{m} \hookrightarrow \mathbb{H}^{n}$ are affine.

It was conjectured in BFS18 and subsequently proved in BC18 that for Heisenberg groups the geodesic linearity property is equivalent to strict convexity of the projection norm, see Definition 2.4. While the point of view presented in BFS18] is purely metric, the essential tools of the proof in [BC18] arise from considering an isometric embedding $\mathbb{R} \hookrightarrow \mathbb{H}^{n}$ as an optimal control problem, and reformulating the first order necessary criterion of the Pontryagin Maximum Principle in the language of convex analysis.

The goal of this paper is to extend the main results of [BFS18] and BC18 to arbitrary Carnot groups of step 2. The central object of study is again the Pontryagin Maximum Principle, from which relevant invariants will be extracted by an asymptotic study of the optimal controls. The main result of the paper is the following:

Theorem 1.1. In every sub-Finsler Carnot group of step 2 with a strictly convex norm, every infinite geodesic is affine.

Corollary 1.2. Let $G$ be a stratified group of step 2 equipped with a homogeneous distance $d$ such that the projection norm of $d$ is strictly convex. Then every infinite geodesic in $(G, d)$ is affine.

The necessity of the strict convexity assumption is a direct consequence of the necessity of strict convexity for linearity of geodesics in 
the normed-space case, see Proposition 5.2. Examples may also be found from the singular geodesics for the non-strictly convex $\ell^{\infty}$ subFinsler norm exhibited in BBLDS17] and ALDS19.

The restriction to step 2 is motivated by the known counterexample in the simplest Carnot group of step 3, the sub-Riemannian Engel group. The complete study of geodesics in the sub-Riemannian Engel group in AS15] gives the first (and to date essentially only) known example of a non-affine infinite geodesic in a sub-Riemannian Carnot group. Note that in general, very little is known about geodesics even in the sub-Riemannian case, see [HLD16, MPV18a, HLD18, $^{\text {BCJ }}{ }^{+} 18$, BdSFPR18 for some recent results.

The proof for Heisenberg groups in BFS18 that the geodesic linearity property of the target implies that all isometric embeddings are affine works also more generally for stratified groups, see Proposition 6.2. Consequently, Corollary 1.2 leads to the analogous rigidity result for arbitrary isometric embeddings:

Theorem 1.3. Let $\left(H, d_{H}\right)$ and $\left(G, d_{G}\right)$ be stratified groups with homogeneous distances such that $G$ has step 2 and the projection norm of $d_{G}$ is strictly convex. Then every isometric embedding $\left(H, d_{H}\right) \hookrightarrow$ $\left(G, d_{G}\right)$ is affine.

It is worth remarking that although there are no explicit restrictions on the domain $\left(H, d_{H}\right)$ in Theorem 1.3, the mere existence of an isometric embedding $\left(H, d_{H}\right) \hookrightarrow\left(G, d_{G}\right)$ implies some restrictions. In particular, Pansu's Rademacher theorem [Pan89] implies that there must exist an injective homogeneous homomorphism $H \rightarrow G$. It follows that $H$ has step at most 2 and rank at most the rank of $G$.

1.1. Structure of the paper. Section 2 presents the relevant definitions that will be used throughout the rest of the paper and some basic lemmas. The main points of interest are properties of blowdowns of geodesics, i.e., geodesics "viewed from afar", and the observations about subdifferentials of convex functions.

Sections 35 are devoted to the proofs of Theorem 1.1 and Corollary 1.2 about infinite geodesics. Section 3 rephrases the classical first order optimality condition of the Pontryagin Maximum Principle in the setting of a step 2 sub-Finsler Carnot group. In the sub-Riemannian case the PMP reduces to a linear ODE for the controls. This is no longer true in the sub-Finsler case, making explicit solution of the system unfeasible. Nonetheless, the PMP has a form (Proposition 3.1) that is well suited to the study of the asymptotic behavior of optimal controls. The key object is the bilinear form $B: V_{1} \times V_{1} \rightarrow \mathbb{R}$.

Section 4 covers the aforementioned asymptotic study. The goal of the section is to study blowdowns of infinite geodesics through the behavior of their controls. Using integral averages of controls, it is 
shown that any blowdown control must in fact be contained in the kernel of the bilinear form $B$.

Section 5 wraps up the proof of Theorem 1.1 using the conclusions of the previous sections. This section is where the strict convexity of the norm is critical. The importance of the assumption is that any linear map has a unique maximum on the ball. By observing that any element of ker $B$ defines an invariant along the corresponding optimal control, the uniqueness is exploited to prove that infinite geodesics must be invariant under blowdowns. Corollary 1.2 follows from the sub-Finsler case by the observation that the length metric associated with a homogeneous norm is always a sub-Finsler metric.

Section [6] covers the proof of Theorem 1.3 about isometric embeddings as a consequence of Corollary 1.2. The link between geodesics and general isometric embeddings arises from considering a foliation by horizontal lines in the domain and studying the induced foliation by infinite geodesics in the image. The affinity of isometric embeddings follows from the observation that two lines are at a sublinear distance from each other if and only if they are parallel.

\section{Preliminaries}

\subsection{Stratified groups and homogeneous distances.}

Definition 2.1. A stratified group is a Lie group $G$ whose Lie algebra has a decomposition $\mathfrak{g}=V_{1} \oplus V_{2} \oplus \cdots \oplus V_{s}$ such that $V_{s} \neq\{0\}$ and $\left[V_{1}, V_{k}\right]=V_{k+1}$ for all $k=1, \ldots, s$, with the convention that $V_{s+1}=\{0\}$. The rank and step of the stratified group $G$ are the integers $r=\operatorname{dim} V_{1}$ and $s$ respectively.

Definition 2.2. A dilation by a factor $\lambda \in \mathbb{R}$ on a stratified group $G$ is the Lie group automorphism $\delta_{\lambda}: G \rightarrow G$ defined for any $X=$ $X_{1}+\cdots+X_{s} \in V_{1} \oplus \cdots \oplus V_{s}$ by

$$
\delta_{\lambda} \exp \left(X_{1}+X_{2}+\cdots+X_{s}\right)=\exp \left(\lambda X_{1}+\lambda^{2} X_{2}+\cdots+\lambda^{s} X_{s}\right) .
$$

Definition 2.3. A homogeneous distance on a stratified group $G$ is a left-invariant distance $d$, which is one-homogeneous with respect to the dilations, i.e., which satisfies

$$
d\left(\delta_{\lambda}(g), \delta_{\lambda}(h)\right)=\lambda d(g, h) \quad \forall \lambda>0, \forall g, h \in G .
$$

\subsection{The projection norm.}

Definition 2.4. Let $G$ be a stratified group and let $d$ be a homogeneous distance on $G$. The projection norm associated with the homogeneous distance $d$ is the function

$$
\|\cdot\|_{d}: V_{1} \rightarrow \mathbb{R}, \quad\|X\|_{d}=d(e, \exp (X)),
$$

where $e$ is the identity element of the group $G$. 
It is not immediate that $\|\cdot\|_{d}$ defines a norm. In the setting of the Heisenberg groups, this is proved in [BFS18, Proposition 2.8]. Their proof works with minor modification for any homogeneous distances in arbitrary stratified groups and is captured in the following lemmas. The triangle inequality of $\|\cdot\|_{d}$ is the only non-trivial part. In order to make use of the triangle inequality of the distance $d$, the following distance estimate is used.

Lemma 2.5. Let $\pi_{V_{1}}: \mathfrak{g}=V_{1} \oplus \cdots \oplus V_{s} \rightarrow V_{1}$ be the projection with respect to the direct sum decomposition. Then

$$
\|X\|_{d} \leq d(e, \exp (X+Y)) \quad \forall X \in V_{1}, \forall Y \in[\mathfrak{g}, \mathfrak{g}],
$$

so the horizontal projection $\pi=\pi_{V_{1}} \circ \log :(G, d) \rightarrow\left(V_{1},\|\cdot\|_{d}\right)$ is a submetry.

Proof. Observe first that for any $X \in V_{1}$ and $Y=Y_{2}+\cdots+Y_{s} \in$ $V_{2} \oplus \cdots \oplus V_{s}=[\mathfrak{g}, \mathfrak{g}]$, and any $n \in \mathbb{N}$, homogeneity and the triangle inequality imply that

$$
\begin{aligned}
n d\left(e, \exp \left(X+\frac{1}{n} Y_{2}+\cdots+\frac{1}{n^{s-1}} Y_{s}\right)\right) & =d(e, \exp (n X+n Y)) \\
& \leq n d(e, \exp (X+Y)) .
\end{aligned}
$$

Continuity of the distance then gives the bound

$$
\begin{aligned}
d(e, \exp (X)) & =\lim _{n \rightarrow \infty} d\left(e, \exp \left(X+\frac{1}{n} Y_{2}+\cdots+\frac{1}{n^{s-1}} Y_{s}\right)\right) \\
& \leq d(e, \exp (X+Y))
\end{aligned}
$$

for any $X \in V_{1}$ and $Y \in[\mathfrak{g}, \mathfrak{g}]$ as claimed.

The previous estimate implies the containment $\pi(B(e, r)) \subset B_{\|\cdot\|_{d}}(0, r)$ for the projection of any ball $B(e, r) \subset G$. On the other hand, Definition 2.4 of the projection norm directly implies the opposite containment

$$
B_{\|\cdot\|_{d}}(0, r)=V_{1} \cap \log B(e, r) \subset \pi(B(e, r)) .
$$

By left-invariance of the distance $d$ it follows that the map $\pi$ is a submetry.

Remark 2.6. The estimate of Lemma 2.5 is in general false for nonhomogeneous left-invariant distances. Examples of the failure may be found by taking any homogeneous metric and tilting the decomposition $V_{1} \oplus[\mathfrak{g}, \mathfrak{g}]$.

Namely, let $d$ be any homogeneous distance on a stratified group $G$ for which the inequality of Lemma 2.5 is strict when $Y \neq 0$, for instance a sub-Riemannian distance. Define a new stratification for the Lie group $G$ by replacing the first layer $V_{1}$ with $\tilde{V}_{1}$, where some vector $X \in V_{1}$ is replaced by $X+Y$ for some central vector $Y \in[\mathfrak{g}, \mathfrak{g}]$. As the Lie brackets and group law are unchanged, $d$ is a left-invariant distance for the resulting stratified group $\tilde{G}$, but is no longer homogeneous due 
to the tilting of the layers. In this way, the notion of projection to the first layer is changed, and the estimate of Lemma 2.5 fails in $(\tilde{G}, d)$ for the vectors $X+Y \in \tilde{V}_{1}$ and $-Y \in[\mathfrak{g}, \mathfrak{g}]$.

Lemma 2.7. The projection norm is a norm.

Proof. Positivity and homogeneity of the projection norm $\|\cdot\|_{d}$ follow immediately from positivity and homogeneity of the homogeneous distance $d$. For the triangle inequality, let $X, X^{\prime} \in V_{1}$ and let $Y \in[\mathfrak{g}, \mathfrak{g}]$ be the element given by the Baker-Campbell-Hausdorff formula such that

$$
\exp (X) \exp \left(X^{\prime}\right)=\exp \left(X+X^{\prime}+Y\right)
$$

Lemma 2.5 gives the bound $\left\|X+X^{\prime}\right\|_{d} \leq d\left(e, \exp \left(X+X^{\prime}+Y\right)\right)$. By the choice of $Y$, the left-invariance and triangle inequality of $d$ conclude the claim:

$$
d\left(e, \exp \left(X+X^{\prime}+Y\right)\right)=d\left(e, \exp (X) \exp \left(X^{\prime}\right)\right) \leq\|X\|_{d}+\left\|X^{\prime}\right\|_{d} .
$$

\subsection{Length structures and sub-Finsler Carnot groups.}

Definition 2.8. Let $(X, d)$ be a metric space. Let $\Omega$ be the space of rectifiable curves of $X$ and let $\ell_{d}: \Omega \rightarrow \mathbb{R}$ be the length functional. For points $x, y \in X$, denote by $\Omega(x, y) \subset \Omega$ the space of all rectifiable curves connecting the points $x$ and $y$. The length metric associated with the metric $d$ is the map $d_{\ell}: X \times X \rightarrow \mathbb{R} \cup\{\infty\}$ defined by

$$
d_{\ell}(x, y):=\inf \left\{\ell_{d}(\gamma): \gamma \in \Omega(x, y)\right\} .
$$

If $d=d_{\ell}$, then the metric $d$ is called a length metric.

See [BBI01, Section 2.3] for further information about length structures induced by metrics. For the purposes of this paper, only the special case of the length metric associated with a homogeneous distance will be relevant. Such a length metric always determines a sub-Finsler Carnot group, see Definition 2.10 and Lemma 5.1.

Definition 2.9. Let $G$ be a stratified group. Denote by $L_{g}: G \rightarrow$ $G$ the left-translation $L_{g}(h)=g h$. An absolutely continuous curve $\gamma:[0, T] \rightarrow G$ is a horizontal curve if $\left(L_{\gamma(t)^{-1}}\right)_{*} \dot{\gamma}(t) \in V_{1}$ for almost every $t \in[0, T]$. The control of a horizontal curve $\gamma$ is its left-trivialized derivative, i.e., the map

$$
u:[0, T] \rightarrow V_{1}, \quad u(t)=\left(L_{\gamma(t)^{-1}}\right)_{*} \dot{\gamma}(t) .
$$

Definition 2.10. A sub-Finsler Carnot group is a stratified group $G$ equipped with a norm $\|\cdot\|: V_{1} \rightarrow \mathbb{R}$. The norm induces a homogeneous distance $d_{S F}$ via the length structure induced by $\|\cdot\|$ over horizontal curves. 
More explicitly, for a horizontal curve $\gamma:[0, T] \rightarrow G$ with control $u:[0, T] \rightarrow V_{1}$, define the length

$$
\ell_{\|\cdot\|}(\gamma)=\int_{0}^{T}\|u(t)\| d t .
$$

For $g, h \in G$, let $\Omega(g, h)$ be the family of all horizontal curves connecting $g$ and $h$. The sub-Finsler distance $d_{S F}$ is defined as

$$
d_{S F}(g, h):=\inf \left\{\ell_{\|\cdot\|}(\gamma): \gamma \in \Omega(g, h)\right\} .
$$

\subsection{Geodesics and blowdowns.}

Definition 2.11. Let $G$ be a stratified group equipped with a homogeneous distance $d$. A geodesic is an isometric embedding $\gamma:[0, T] \rightarrow$ $(G, d)$. That is, a geodesic satisfies

$$
d(\gamma(t), \gamma(s))=|t-s| \quad \forall t, s \in[0, T] .
$$

In the proof of Theorem 1.3 it will be convenient to consider also curves which preserve distances up to a constant factor. A curve $\gamma:[0, T] \rightarrow(G, d)$ for which there exists some constant $C>0$ such that

$$
d(\gamma(t), \gamma(s))=C|t-s| \quad \forall t, s \in[0, T]
$$

will be called a geodesic with speed $C$.

Lemma 2.12. Let $\gamma:[0, \infty) \rightarrow G$ be a horizontal curve with control $u:[0, \infty) \rightarrow V_{1}$ and let $\lambda>0$ be a dilating factor. Then the dilated and reparametrized curve

$$
\gamma_{\lambda}:[0, \infty) \rightarrow G, \quad \gamma_{\lambda}(t):=\delta_{1 / \lambda} \gamma(\lambda t)
$$

has the control

$$
u_{\lambda}:[0, \infty) \rightarrow V_{1}, \quad u_{\lambda}(t):=u(\lambda t)
$$

Proof. Since the dilations are group homomorphisms, the claim follows directly by the chain rule and Definition 2.9 of a control:

$$
\frac{d}{d t} \gamma_{\lambda}(t)=\left(\delta_{1 / \lambda}\right)_{*} \frac{d}{d t} \gamma(\lambda t)=\left(\delta_{1 / \lambda}\right)_{*}\left(L_{\gamma(\lambda t)}\right)_{*} u(\lambda t) \lambda=\left(L_{\gamma_{\lambda}(t)}\right)_{*} u_{\lambda}(t)
$$

Definition 2.13. Let $\gamma:[0, \infty) \rightarrow G$ be a horizontal curve. Suppose for some sequence of scales $\lambda_{k} \rightarrow \infty$ the pointwise limit

$$
\tilde{\gamma}:[0, \infty) \rightarrow G, \quad \tilde{\gamma}(t)=\lim _{k \rightarrow \infty} \gamma_{\lambda_{k}}(t)=\lim _{k \rightarrow \infty} \delta_{1 / \lambda_{k}} \gamma\left(\lambda_{k} t\right)
$$

exists. Such a curve $\tilde{\gamma}$ is called a blowdown of the curve $\gamma$ along the sequence of scales $\lambda_{k}$.

Remark 2.14. If the curve $\gamma$ is $L$-Lipschitz, then the curves $\gamma_{\lambda}$ are also all $L$-Lipschitz. Hence by Arzelà-Ascoli, up to taking a subsequence a blowdown along a sequence of scales will always exist. 
Lemma 2.15. Let $\gamma:[0, \infty) \rightarrow G$ be an infinite geodesic and let $\tilde{\gamma}=$ $\lim _{k \rightarrow \infty} \gamma_{\lambda_{k}}$ be any blowdown of the curve $\gamma$. Let $u$ and $\tilde{u}$ be the controls of the curves $\gamma$ and $\tilde{\gamma}$ respectively. Then

(i) The curve $\tilde{\gamma}$ is an infinite geodesic.

(ii) Up to taking a subsequence, the dilated controls $u_{\lambda_{k}}$ converge to the control $\tilde{u}$ in $\mathrm{L}_{l o c}^{2}\left([0, \infty) ; V_{1}\right)$.

Proof. (i). The curve $\tilde{\gamma}$ is a geodesic as the pointwise limit of geodesics.

(ii). The claim follows from [MPV18b, Remark 3.13]. The point is that by weak compactness of closed balls in $\mathrm{L}_{\text {loc }}^{2}\left([0, \infty) ; V_{1}\right)$ there exists a weakly convergent subsequence $u_{\lambda} \rightarrow v$ to some $v \in \mathrm{L}_{\text {loc }}^{2}\left([0, \infty) ; V_{1}\right)$. The definitions of control and weak convergence imply that $v$ is a control for $\tilde{\gamma}$, so in particular $\tilde{u}(t)=v(t)$ for almost every $t$. Finally, the geodesic assumption implies that $\|u(t)\| \equiv 1 \equiv\|\tilde{u}(t)\|$, so the weak convergence is upgraded to strong convergence $u_{\lambda} \rightarrow \tilde{u}$ in $\mathrm{L}_{\text {loc }}^{2}\left([0, \infty) ; V_{1}\right)$.

Lemma 2.16. Let $G$ be a sub-Finsler Carnot group with a strictly convex norm and let $\gamma:[0, \infty) \rightarrow G$ be an infinite geodesic. Then there exists a sequence $\lambda_{k} \rightarrow \infty$ such that the blowdown $\tilde{\gamma}=\lim _{k \rightarrow \infty} \gamma_{\lambda_{k}}$ is affine.

Proof. If the geodesic $\gamma$ is itself affine, then the claim is immediate. Suppose then that $\gamma$ is not affine, i.e., not a left translation of a one parameter subgroup. In particular, the geodesic $\gamma$ has non-constant control. Hence the horizontal projection $\pi \circ \gamma:[0, \infty) \rightarrow G /[G, G]$ has non-constant derivative, and is also not affine. Since $G /[G, G]$ is a normed space with a strictly convex norm, the projection curve $\pi \circ \gamma$ cannot be a geodesic. Then [HLD18, Theorem 1.4] states that there exists a Carnot subgroup $H<G$ of lower rank such that all blowdowns of the geodesic $\gamma$ are contained in $H$.

Let the curve $\beta:[0, \infty) \rightarrow H<G$ be any blowdown. By Lemma2.15)(i), $\beta$ is a geodesic. If $\beta$ is also not affine, then iterating the above there exists a Carnot subgroup $K<H<G$ of even lower rank such that all blowdowns of $\beta$ are in $K$. Blowdowns of the geodesic $\beta$ are also blowdowns of the geodesic $\gamma$ by a diagonal argument, so the claim follows by induction, since a Carnot subgroup of rank 1 is just a one parameter subgroup.

2.5. Subdifferentials. In this section, let $V$ be some fixed finite dimensional vector space and let $E: V \rightarrow \mathbb{R}$ be a convex continuous function. In the application in Section 5 , the space $V$ will be the horizontal layer $V_{1} \subset \mathfrak{g}$, and the convex function of interest will be a squared norm $\frac{1}{2}\|\cdot\|^{2}$. 
Definition 2.17. A linear function $a: V \rightarrow \mathbb{R}$ is a subdifferential of the function $E$ at a point $Y \in V$ if

$$
a(X-Y) \leq E(X)-E(Y) \quad \forall X \in V .
$$

The collection of all subdifferentials $a$ at a point $Y \in V$ is denoted $\partial E(Y) \subset V^{*}$.

The following lemmas condense the properties of convex functions and their subdifferentials that will be relevant for the article. They will be utilized in the proof of Theorem 1.1 in Section 5. The first lemma is the continuity and compactness of subdifferentials.

Lemma 2.18. Let $Y_{k} \rightarrow Y \in V$ be a converging sequence and let $a_{k} \in$ $\partial E\left(Y_{k}\right)$. Then there exists a converging subsequence $a_{k} \rightarrow a \in \partial E(Y)$.

Proof. [Roc70, Theorem 24.7] shows (among other things) that since the set of points $\mathcal{S}=\left\{Y_{i}: i \in \mathbb{N}\right\} \cup\{Y\}$ is compact, the family of subdifferentials

$$
\partial E(\mathcal{S}):=\bigcup_{X \in \mathcal{S}}\{a \in \partial E(X)\}
$$

is also compact. Hence there exists a converging subsequence $a_{k} \rightarrow$ $a \in \partial E(\mathcal{S})$. The claim is concluded by [Roc70, Theorem 24.4], which shows that the convergences $Y_{k} \rightarrow Y$ and $a_{k} \rightarrow a$ with $a_{k} \in \partial E\left(Y_{k}\right)$ imply that $a \in \partial E(Y)$.

The second lemma is a simple estimate on a subdifferential of the squared norm.

Lemma 2.19. Let $\|\cdot\|$ be a norm on $V$ and let $a: V \rightarrow \mathbb{R}$ be a subdifferential of the squared norm $E=\frac{1}{2}\|\cdot\|^{2}$ at a point $Y \in V$. Then $|a(X)| \leq\|X\|\|Y\|$ for all $X \in V$, and $a(Y)=\|Y\|^{2}$.

Proof. For any points $X, Y \in V$ and any $\epsilon>0$, the subdifferential condition $a \in \partial E(Y)$ implies that

$$
\begin{aligned}
\epsilon a(X) & =a(Y+\epsilon X-Y) \leq E(Y+\epsilon X)-E(Y) \\
& \leq \frac{1}{2}\left((\|Y\|+\epsilon\|X\|)^{2}-\|Y\|^{2}\right)=\epsilon\|X\|\|Y\|+\frac{1}{2} \epsilon^{2}\|X\|^{2} .
\end{aligned}
$$

Letting $\epsilon \rightarrow 0$ proves the bound $a(X) \leq\|X\|\|Y\|$. Repeating the same consideration for $-X$, gives the opposite bound $-a(X) \leq\|X\|\|Y\|$.

For the equality $a(Y)=\|Y\|^{2}$, let $\epsilon>0$, and observe that a similar computation as before shows that

$$
\begin{aligned}
-\epsilon a(Y) & =a((1-\epsilon) Y-Y) \leq E((1-\epsilon) Y)-E(Y) \\
& =\frac{1}{2}\left((1-\epsilon)^{2}-1\right)\|Y\|^{2}=\left(-\epsilon+\frac{1}{2} \epsilon^{2}\right)\|Y\|^{2} .
\end{aligned}
$$

That is, $a(Y) \geq\left(1-\frac{1}{2} \epsilon\right)\|Y\|^{2}$. The limit as $\epsilon \rightarrow 0$ and the previous upper bound prove the claim. 


\section{Step 2 sub-Finsler Pontryagin Maximum Principle}

In this section, the Pontryagin Maximum Principle will be rephrased in a convenient form for the purposes of Theorem 1.1. The precise statement to be proved is the following:

Proposition 3.1 (Step 2 sub-Finsler PMP.). Let $G$ be a step 2 subFinsler Carnot group with an arbitrary norm $\|\cdot\|: V_{1} \rightarrow \mathbb{R}$ and let $0 \leq T \leq \infty$. If $u:[0, T] \rightarrow V_{1}$ is the control of a geodesic, then there exists an absolutely continuous curve $a:[0, T] \rightarrow V_{1}^{*}$ and a skewsymmetric bilinear form $B: V_{1} \times V_{1} \rightarrow \mathbb{R}$ such that

(i) At almost every $t \in[0, T]$, the curve a has the derivative

$$
\frac{d}{d t} a(t) Y=B(u(t), Y) \quad \forall Y \in V_{1} .
$$

(ii) At almost every $t \in[0, T]$, the linear map $a(t): V_{1} \rightarrow \mathbb{R}$ is a subdifferential of the squared norm $\frac{1}{2}\|\cdot\|^{2}$ at the point $u(t) \in V_{1}$.

Remark 3.2. Up to changing the optimal control $u$ on a set of measure zero, the subdifferential condition (ii) may be taken to hold for all $t \in[0, T]$.

Namely, if condition (ii) holds on a subset $I \subset[0, T]$ of full measure, for any $t \in[0, T] \backslash I$, pick any converging sequence $I \ni t_{k} \rightarrow t$ such that the limit $\lim _{k \rightarrow \infty} u\left(t_{k}\right)$ exists, and redefine $u(t)=\lim _{k \rightarrow \infty} u\left(t_{k}\right)$. By the continuity of subdifferentials given by Lemma 2.18, it follows that $a(t)$ is a subdifferential of the squared norm at the point $u(t)$.

Remark 3.3. In the sub-Riemannian case, the squared norm $\frac{1}{2}\|\cdot\|^{2}$ is differentiable at every point, and the unique subdifferential is the inner product $a(t)=\langle u(t), \cdot\rangle$. The derivative condition (i) then gives the usual linear ODE of controls in the implicit form

$$
\langle\dot{u}(t), Y\rangle=\frac{d}{d t}\langle u(t), Y\rangle=B(u(t), Y) \quad \forall Y \in V_{1} .
$$

3.1. General statement of the PMP. For the rest of Section 3 , let $G$ be a fixed sub-Finsler Carnot group of step 2 with an arbitrary norm $\|\cdot\|: V_{1} \rightarrow \mathbb{R}$, and let $u:[0, T] \rightarrow V_{1}$ be the control of a geodesic $\gamma:[0, T] \rightarrow G$.

Consider first the finite time $T<\infty$ case. By Definition 2.10 of the sub-Finsler distance, the control $u$ minimizes the length functional $\int_{0}^{T}\|u(t)\| d t$ among all controls defining curves with the same endpoints as $\gamma$. Since a geodesic has by definition constant speed, it follows that $u$ is also a minimizer of the energy functional $\frac{1}{2} \int_{0}^{T}\|u(t)\|^{2} d t$.

Define the left-trivialized Hamiltonian

$$
h: V_{1} \times \mathbb{R} \times \mathfrak{g}^{*} \rightarrow \mathbb{R}, \quad h(u, \xi, \lambda)=\lambda(u)+\frac{1}{2} \xi\|u\|^{2} .
$$

By the Pontryagin Maximum Principle as presented in [AS04, Theorem 12.10], the control $u:[0, T] \rightarrow V_{1}$ can minimize the energy $\frac{1}{2} \int_{0}^{T}\|u(t)\|^{2} d t$ 
only if there is an everywhere non-zero absolutely continuous dual curve $t \mapsto(\xi, \lambda(t)) \in \mathbb{R} \times T_{\gamma(t)}^{*} G$ such that

$$
\begin{aligned}
\xi & \leq 0 \\
\dot{\lambda} & =\vec{h}_{u(t), \xi}(\lambda) \quad \text { a.e. } t \in[0, T], \\
h_{u(t), \xi}(\lambda(t)) & \geq h_{v, \xi}(\lambda(t)) \quad \forall v \in V_{1} \quad \text { a.e. } t \in[0, T] .
\end{aligned}
$$

Here $h_{v, \xi}$ and $\vec{h}_{v, \xi}$, for $v \in V_{1}$, are the left-invariant Hamiltonian and the associated Hamiltonian vector field respectively.

More explicitly, $h_{v, \xi}: T^{*} G \rightarrow \mathbb{R}$ is the function defined from the left-trivialized Hamiltonian (1) in the natural way by

$$
h_{v, \xi}(\lambda)=h\left(v, \xi, L_{g}^{*} \lambda\right), \quad \forall \lambda \in T_{g}^{*} G,
$$

and $\vec{h}_{v, \xi}$ is the Hamiltonian vector field associated with the left-invariant Hamiltonian $h_{v, \xi}$ by duality through the canonical symplectic form on the cotangent bundle, see ABB19, Section 4] for more details within the context of the PMP in the sub-Riemannian setting.

Observe that if $(\xi, \lambda(t))$ is a dual curve satisfying the conditions (2) -(4) of the PMP, then also any scalar multiple $(C \xi, C \lambda(t))$ for any $C>0$ satisfies the conditions (2) -(4) of the PMP. This observation allows the infinite time case $T=\infty$ to be handled as a limit of the finite time case. Namely, if $u:[0, \infty) \rightarrow V_{1}$ is the control of a geodesic, then all its finite restrictions $\left.u\right|_{[0, k]}:[0, k] \rightarrow V_{1}$ for $k \in \mathbb{N}$ are also controls of geodesics, so by the above they have corresponding dual curves $t \mapsto$ $\left(\xi_{k}, \lambda_{k}(t)\right)$. By taking suitable rescalings of the $\left(\xi_{k}, \lambda_{k}\right)$, there exists a non-zero limit $\left(\xi_{\infty}, \lambda_{\infty}\right)$, which then satisfies the conditions (2)-(44) of the PMP on the entire interval $[0, \infty)$.

Condition (2) is a binary condition $\xi=0$ or $\xi \neq 0$. The case $\xi=0$ is the case of an abnormal control $u$, and may be ignored in the step 2 setting, since the second order necessary criterion of the Goh condition (see e.g. [AS04, Section 20]) implies that there are no strictly abnormal extremals in step 2. By rescaling $(\xi, \lambda)$ it therefore suffices to consider the normal case $\xi=-1$.

3.2. The PMP in left-trivialized coordinates. Let $X_{1}, \ldots, X_{r}$ be a basis of $V_{1}$. Fix a basis $X_{r+1}, \ldots, X_{n}$ for $V_{2}=\left[V_{1}, V_{1}\right]$ by choosing a maximal linearly independent subset of the Lie brackets $\left\{\left[X_{i}, X_{j}\right]: 1 \leq\right.$ $i<j \leq r\}$. By an abuse of notation, denote also by $X_{1}, \ldots, X_{n}$, the corresponding left-invariant frame of $T G$. Let $\theta_{1}, \ldots, \theta_{n}$ be the dual left-invariant frame of $T^{*} G$. Writing the curve $\lambda(t)$ in left-trivialized coordinates as

$$
\lambda(t)=\sum_{i=1}^{n} \lambda_{i}(t) \theta_{i}(\gamma(t))
$$


the Hamiltonian ODE (3) in the normal case $\xi=-1$ takes the simpler form

$$
\dot{\lambda}_{i}(t)=\lambda(t)\left(\left[\sum_{j=1}^{r} u_{j}(t) X_{j}, X_{i}\right](\gamma(t))\right), \quad i=1, \ldots, n,
$$

see [AS04, Section 18.3] for the explicit computation.

Proof of Proposition 3.1. The curve $a:[0, T] \rightarrow V_{1}^{*}$ will be given by restricting the linear map

$$
a(t):=\left(L_{\gamma(t)}\right)^{*} \lambda(t): \mathfrak{g} \rightarrow \mathbb{R}
$$

to $V_{1}$. The skew-symmetric bilinear form $B: V_{1} \times V_{1} \rightarrow \mathbb{R}$ will be given by

$$
B(X, Y):=a(t)[X, Y] .
$$

The curve $a(t)$ of (6) has in the left-invariant frame the same coefficients as the curve $\lambda(t)$, i.e., the coefficients of $a(t)=\sum_{i=1}^{n} a_{i}(t) \theta_{i}(e)$ are exactly $a_{i}=\lambda_{i}$. Left-translating the Hamiltonian ODE (5) to the identity shows that for almost every $t \in[0, T]$, the components of the curve have the derivatives

$$
\dot{a}_{i}(t)=\frac{d}{d t} \lambda_{i}(t)=a(t)\left[u(t), X_{i}\right], \quad i=1, \ldots, n .
$$

By the step 2 assumption, $\left[u(t), X_{i}\right]=0$ for all the vertical components $i=r+1, \ldots, n$, so the vertical coefficients $a_{r+1}, \ldots, a_{n}$ are all constant. Therefore $a(t)[X, Y]=\sum_{i=r+1}^{n} a_{i} \theta_{i}([X, Y])$ is constant in $t$. That is, the expression (7) defines a unique bilinear form $B$ independent from $t$.

Writing the system (8) in terms of the bilinear form $B$, the remaining non-trivial equations are exactly

$$
\dot{a}_{i}(t)=a(t)\left[u(t), X_{i}\right]=B\left(u(t), X_{i}\right), \quad i=1, \ldots, r .
$$

The derivative condition 3.1](i) follows by linearity, as for an arbitrary vector $Y=y_{1} X_{1}+\cdots+y_{r} X_{r} \in V_{1}$, the above implies that

$$
\frac{d}{d t} a(t) Y=\frac{d}{d t} \sum_{i=1}^{n} a_{i}(t) y_{i}=\sum_{i=1}^{n} B\left(u(t), X_{i}\right) y_{i}=B(u(t), Y) .
$$

The subdifferential condition 3.1](ii) for the linear functions $a(t)$ follows from rephrasing the maximality condition (44). Namely, expanding out the explicit expressions of the normal Hamiltonians $h_{u(t),-1}$ and $h_{v,-1}$ from (1) and reorganizing terms, the maximality condition (4) is equivalently stated as

$$
a(t) v-a(t) u(t) \leq \frac{1}{2}\|v\|^{2}-\frac{1}{2}\|u(t)\|^{2} \quad \forall v \in V_{1} \quad \text { a.e. } t \in[0, T] .
$$

This is exactly Definition 2.17 stating that the linear function $a(t)$ is a subdifferential of the squared norm $\frac{1}{2}\|\cdot\|^{2}$ at the point $u(t) \in V_{1}$. 


\section{Asymptotic Behavior of CONTROLS}

In this section, let $u:[0, \infty) \rightarrow V_{1}$ be a fixed control satisfying the PMP [3.1. Let $a:[0, \infty) \rightarrow V_{1}^{*}$ be the associated curve of subdifferentials and let $B: V_{1} \times V_{1} \rightarrow \mathbb{R}$ be the associated bilinear form.

Lemma 4.1. For every vector $X \in V_{1}$,

$$
\lim _{T \rightarrow \infty} B\left(f_{0}^{T} u(t) d t, X\right)=0
$$

Proof. Fix an arbitrary vector $X \in V_{1}$. Bilinearity of the map $B$ implies that

$$
B\left(f_{0}^{T} u(t) d t, X\right)=\frac{1}{T} \int_{0}^{T} B(u(t), X) d t .
$$

Since the curve $a$ is absolutely continuous, the derivative condition PMP 3.1](i) implies that

$$
\int_{0}^{T} B(u(t), X)=\int_{0}^{T} \frac{d}{d t} a(t) X=a(T) X-a(0) X .
$$

By the subdifferential condition PMP 3.1](ii), for almost every $T$, the linear map $a(T)$ is a subdifferential of the squared norm $\frac{1}{2}\|\cdot\|^{2}$ at the point $u(T)$. Since $\|u(T)\| \equiv 1$ is constant, continuity of the curve $a$ and Lemma 2.19 imply the bound $|a(T) X| \leq\|X\|$ for every $T \in[0, \infty)$. The identities (9) and (10) then imply the desired conclusion that

$$
\lim _{T \rightarrow \infty}\left|B\left(f_{0}^{T} u(t) d t, X\right)\right| \leq \lim _{T \rightarrow \infty} \frac{2}{T}\|X\|=0 .
$$

Lemma 4.2. Let $\lambda_{k} \rightarrow \infty$ be a diverging sequence and let $u_{\lambda_{k}}(t)=$ $u\left(\lambda_{k} t\right)$ be the corresponding dilated controls. If $u_{\lambda_{k}} \rightarrow \tilde{u}$ in $\mathrm{L}_{l o c}^{2}\left([0, \infty) ; V_{1}\right)$, then $\tilde{u}(t) \in \operatorname{ker} B$ for almost every $t \in[0, \infty)$.

Proof. By the Lebesgue differentiation theorem it suffices to prove that $f_{a}^{b} \tilde{u}(t) d t \in \operatorname{ker} B$ for any $0 \leq a<b<\infty$.

Fix $0 \leq a<b<\infty$. By assumption $u_{\lambda_{k}} \rightarrow \tilde{u}$ in $\mathrm{L}^{2}\left([a, b] ; V_{1}\right)$, so there exists some error term $\epsilon: \mathbb{N} \rightarrow V_{1}$ with $\lim _{k \rightarrow \infty} \epsilon(k)=0$ such that

$$
f_{a}^{b} \tilde{u}(t) d t=f_{a}^{b} u\left(\lambda_{k} t\right) d t+\epsilon(k)=\int_{a \lambda_{k}}^{b \lambda_{k}} u(t) d t+\epsilon(k) .
$$

The right-hand integral average can further be expressed as a difference of integral averages as

$$
f_{a \lambda_{k}}^{b \lambda_{k}} u(t) d t=\frac{b}{b-a} \cdot f_{0}^{b \lambda_{k}} u(t) d t-\frac{a}{b-a} \cdot f_{0}^{a \lambda_{k}} u(t) d t .
$$

Lemma 4.1 implies that for any $X \in V_{1}$

$$
\lim _{k \rightarrow \infty} B\left(f_{0}^{b \lambda_{k}} u(t) d t, X\right)=\lim _{k \rightarrow \infty} B\left(f_{0}^{a \lambda_{k}} u(t) d t, X\right)=0 .
$$


Combining the identities (11) and (12) and using bilinearity of $B$ then implies that $B\left(f_{a}^{b} \tilde{u}(t) d t, X\right)=0$. Since the vector $X \in V_{1}$ was arbitrary, this proves the desired claim that $f_{a}^{b} \tilde{u}(t) d t \in \operatorname{ker} B$.

\section{Affinity of infinite GeOdesics}

5.1. Sub-Finsler Carnot groups. The proof of Theorem 1.1 will now be concluded. The key ingredients are the sub-Finsler PMP [3.1, the knowledge of asymptotic behavior of blowdown controls from Lemma 4.2, and the convex analysis arguments from Subsection 2.5.

Proof of Theorem 1.1. Let $\gamma:[0, \infty) \rightarrow G$ be an infinite geodesic and let $u:[0, \infty) \rightarrow V_{1}$ be its control. Let $a:[0, \infty) \rightarrow V_{1}^{*}$ be the curve of subdifferentials of the squared norm $\frac{1}{2}\|\cdot\|^{2}$ and let $B: V_{1} \times V_{1} \rightarrow \mathbb{R}$ be the skew-symmetric bilinear form given by the PMP 3.1.

By Lemma 2.16, there exists a sequence $\lambda_{k} \rightarrow \infty$ such that the blowdown $\tilde{\gamma}=\lim _{k \rightarrow \infty} \delta_{1 / \lambda_{k}} \circ \gamma \circ \delta_{\lambda_{k}}:[0, \infty) \rightarrow G$ is affine, i.e., a left translation of a one parameter subgroup. By Lemma 2.15, taking a subsequence if necessary, the dilated controls $u_{\lambda_{k}}(t)=u\left(\lambda_{k} t\right)$ converge in $\mathrm{L}_{\text {loc }}^{2}\left([0, \infty) ; V_{1}\right)$ to the control $\tilde{u}$ of the curve $\tilde{\gamma}$. Since the curve $\tilde{\gamma}$ is affine, the control $\tilde{u}$ is constant. That is, there exists a constant vector $Y \in V_{1}$, which for almost every $t \in[0, \infty)$ is the limit

$$
Y=\tilde{u}(t)=\lim _{k \rightarrow \infty} u\left(\lambda_{k} t\right) .
$$

By Lemma 4.2, $Y \in \operatorname{ker} B$, so the derivative condition PMP 3.1][(i) implies that the curve $t \mapsto a(t) Y$ is constant $a(t) Y \equiv: C$.

Fix any $t \in[0, \infty)$ such that the limit (13) holds. By Lemma 2.18, up to taking a further subsequence, the subdifferentials $a\left(\lambda_{k} t\right)$ of the squared norm $\frac{1}{2}\|\cdot\|^{2}$ at the points $u\left(\lambda_{k} t\right)$ converge to a subdifferential $\tilde{a}: V_{1} \rightarrow \mathbb{R}$ of the squared norm $\frac{1}{2}\|\cdot\|^{2}$ at the point $Y$. Moreover, since $a(t) Y \equiv C$ is constant, also the limit evaluates to $\tilde{a} Y=C$. Applying Lemma 2.19 for the subdifferential $\tilde{a}$ shows that $C=\tilde{a} Y=$ $\|Y\|^{2}$. Similarly applying Lemma 2.19 for the subdifferential $a(t)$ shows that $a(t) u(t)=\|u(t)\|^{2}$. Since the curves $\gamma$ and $\tilde{\gamma}$ are both geodesics, $\|u(t)\|=1=\|Y\|$, so combining all of the above gives the equality

$$
a(t) Y=1=a(t) u(t) .
$$

Consequently for any convex combination $X \in V_{1}$ of $u(t)$ and $Y$, Lemma 2.19 implies that

$$
1=a(t) X \leq\|X\|\|u(t)\|=\|X\| .
$$

By strict convexity of the norm this is only possible when $u(t)=Y$.

Repeating the same argument at all the times $t$ satisfying the limit (13), it follows that $u(t)=Y$ for almost every $t \in[0, \infty)$, so the geodesic $\gamma$ is affine. 
5.2. Arbitrary homogeneous distances. The proof of Corollary 1.2 about infinite geodesics for arbitrary homogeneous distances follows from the sub-Finsler case by passing to the induced length metric. The relevant properties are captured in the next lemma.

Lemma 5.1. Let $(G, d)$ be a stratified group equipped with a homogeneous distance $d$ and let $d_{\ell}$ be the length metric of $d$. Then

(i) $\left(G, d_{\ell}\right)$ is a sub-Finsler Carnot group.

(ii) All geodesics of $(G, d)$ are also geodesics of $\left(G, d_{\ell}\right)$.

(iii) The projection norm of $d$ is the sub-Finsler norm of $d_{\ell}$.

Proof. (i). In [LD15, Theorem 1.1] sub-Finsler Carnot groups are characterized as the only geodesic metric spaces that are locally compact, isometrically homogeneous, and admit a dilation. Therefore it suffices to verify that the length metric associated with a homogeneous distance satisfies these properties.

The claims of isometric homogeneity and admitting a dilation follow directly from the corresponding properties of the metric $d$. Namely, since left-translations are isometries of the metric $d$, they preserve the length of curves, and hence are also isometries of the length metric $d_{\ell}$. Similarly since dilations scale the length of curves linearly, they are dilations for the length metric $d_{\ell}$.

Finiteness of the length metric $d_{\ell}$ follows from the stratification assumption: each element $g \in G$ can be written as a product of elements in $\exp \left(V_{1}\right)$ and the horizontal lines $t \mapsto \exp (t X)$ are all geodesics. Therefore concatenation of suitable horizontal line segments defines a finite length curve from the identity $e$ to any desired point $g$. It follows that the length metric $d_{\ell}$ determines a well defined homogeneous distance on $G$, so by [LDR19, Proposition 2.26] it induces the manifold topology of $G$. In particular $\left(G, d_{\ell}\right)$ is a boundedly compact length space, so it is a geodesic metric space (see BBI01, Corollary 2.5.20]). Applying [LD15, Theorem 1.1] shows that $\left(G, d_{\ell}\right)$ is a sub-Finsler Carnot group.

(ii). The lengths of all rectifiable curves in the original metric $d$ and its associated length metric $d_{\ell}$ always agree (see [BBI01, Proposition 2.3.12]). In particular, the claim that the geodesics of $(G, d)$ are geodesics of $\left(G, d_{\ell}\right)$ follows.

(iii). The horizontal projection $\pi:(G, d) \rightarrow V_{1}$ is a submetry both for the sub-Finsler norm $\|\cdot\|_{S F}$ (by definition) and for the projection norm $\|\cdot\|_{d}$ (by Lemma 2.5). Hence the norms $\|\cdot\|_{S F}$ and $\|\cdot\|_{d}$ have exactly the same balls, so $\|\cdot\|_{S F}=\|\cdot\|_{d}$.

Proof of Corollary 1.2. Let $(G, d)$ be a stratified group of step 2 equipped with a homogeneous distance $d$ whose projection norm is strictly convex, and let $\gamma:[0, \infty) \rightarrow(G, d)$ be an infinite geodesic. 
Let $d_{\ell}$ be the length-metric associated with $d$. By Lemma 5.1](i) and (ii), the curve $\gamma$ is also a geodesic of $(G,\|\cdot\|)$, where $\|\cdot\|: V_{1} \rightarrow \mathbb{R}$ is the sub-Finsler norm of the sub-Finsler metric $d_{\ell}$. Moreover by Lemma 5.1](iii) the norm $\|\cdot\|=\|\cdot\|_{d}$ is by assumption strictly convex.

Consequently by Theorem 1.1, the geodesic $\gamma$ is affine.

The necessity of the strict convexity assumption is an immediate consequence of the classical case of normed spaces by the following simple lifting argument.

Proposition 5.2. Let $G$ be a stratified group equipped with an arbitrary homogeneous distance $d$. If the projection norm of $d$ is not strictly convex, then there exist an infinite geodesic $\gamma: \mathbb{R} \rightarrow G$ which is not affine.

Proof. If the projection norm $\|\cdot\|_{d}: V_{1} \rightarrow \mathbb{R}$ is not strictly convex, then there exists a non-linear geodesic $\beta: \mathbb{R} \rightarrow V_{1}$. For example, if the norm $\|X+c Y\|_{d}$ is constant for $-\epsilon \leq c \leq \epsilon$, then the curve $\beta(t)=$ $t X+\epsilon \sin (t) Y$ is an infinite geodesic.

By Lemma 2.5, the projection $\pi:(G, d) \rightarrow\left(V_{1},\|\cdot\|\right)$ is a submetry, so the geodesic $\beta: \mathbb{R} \rightarrow V_{1}$ lifts to an infinite geodesic $\gamma: \mathbb{R} \rightarrow G$. Since the projection is a homomorphism and the geodesic $\beta$ is not affine, neither is the geodesic $\gamma$.

\section{AfFinity of isometric EMBEDDings}

Theorem 1.3 about isometric embeddings being affine follows from Corollary 1.2 by an abstraction of the argument of BFS18, Theorem 4.1]. The abstract version of their statement is Proposition 6.2. The key link between the metric and algebraic properties is the following simple lemma stating that the distance between two lines grows sublinearly if and only if the lines are parallel.

Lemma 6.1. Let $(G, d)$ be a stratified group with a homogeneous distance. Then for all points $g, h \in G$ and all vectors $X, Y \in V_{1}$

$$
d(g \exp (t X), h \exp (t Y))=o(t) \text { as } t \rightarrow \infty \Longleftrightarrow X=Y .
$$

Proof. Consider dilations by $1 / t$. Since dilations are homomorphisms, continuity of the distance gives the limit

$$
\begin{aligned}
\lim _{t \rightarrow \infty} \frac{d(g \exp (t X), h \exp (t Y))}{t} & =\lim _{t \rightarrow \infty} d\left(\delta_{1 / t}(g) \exp (X), \delta_{1 / t}(h) \exp (Y)\right) \\
& =d(\exp (X), \exp (Y))
\end{aligned}
$$

Proposition 6.2. Let $\left(H, d_{H}\right)$ and $\left(G, d_{G}\right)$ be stratified groups with homogeneous distances such that all infinite geodesics in $G$ are affine. Then every isometric embedding $\left(H, d_{H}\right) \hookrightarrow\left(G, d_{G}\right)$ is affine.

Proof. Let $\varphi:\left(H, d_{H}\right) \hookrightarrow\left(G, d_{G}\right)$ be an isometric embedding. Since left-translations are isometries, it suffices to consider the case when the 
map $\varphi$ preserves the identity element, and prove that such an isometric embedding is a homomorphism.

Consider an arbitrary point $h \in H$ and a horizontal vector $X \in V_{1}^{H}$. The horizontal line $t \mapsto h \exp (t X)$ is an infinite geodesic with speed $\|X\|_{H}$ through the point $h \in H$. The image of the line under the isometric embedding $\varphi$ is an infinite geodesic in the group $G$ through the point $\varphi(h)$ with exactly the same speed. By assumption all infinite geodesics in the group $G$ are horizontal lines, so there exists some vector $Y \in V_{1}^{G}$ (a priori depending on the point $h$ and the vector $X$ ) with $\|X\|_{H}=\|Y\|_{G}$ such that

$$
\varphi(h \exp (t X))=\varphi(h) \exp (t Y) \quad \forall t \in \mathbb{R} .
$$

Consider then the two parallel infinite geodesics $t \mapsto \exp (t X)$ and $t \mapsto h \exp (t X)$ with speed $\|X\|_{H}$. Repeating the previous consideration, since the map $\varphi$ was assumed to preserve the identity, there exists another horizontal direction $Z \in V_{1}^{G}$ such that $\varphi(\exp (t X))=\exp (t Z)$. By Lemma 6.1, the distance between the two lines in the group $H$ grows sublinearly. Since the map $\varphi$ is an isometric embedding, also the distance between the image lines in the group $G$ grows sublinearly. Hence applying Lemma 6.1 in the converse direction implies that $Y=Z$. That is, the vector $Y \in V_{1}^{G}$ does not depend on the point $h \in H$, only on the vector $X \in V_{1}^{H}$.

The above shows that there is a well defined map $\varphi_{*}: V_{1}^{H} \rightarrow V_{1}^{G}$ such that $\varphi(h \exp (X))=\varphi(h) \exp \left(\varphi_{*} X\right)$. In particular,

$$
\varphi\left(h_{1} h_{2}\right)=\varphi\left(h_{1}\right) \varphi\left(h_{2}\right) \quad \forall h_{1} \in H \forall h_{2} \in \exp \left(V_{1}^{H}\right) .
$$

Since the group $H$ is stratified, the subset $\exp \left(V_{1}^{H}\right)$ generates the entire group $H$. That is, any element $h \in H$ can be written as a finite product of elements in $\exp \left(V_{1}^{H}\right)$. Applying the identity (14) repeatedly using such decompositions shows that the map $\varphi$ is a homomorphism.

Theorem 1.3 follows directly by combining the statements of Corollary 1.2 and Proposition 6.2 .

Acknowledgements. The author wishes to thank Enrico Le Donne and Yuri Sachkov for helpful discussions on the control viewpoint to infinite geodesics that paved the way to the conclusion of the main proof. The author also wishes to thank Ville Kivioja for his help during the outset of the project in the study of the known results and their abstractions.

\section{REFERENCES}

[ABB19] Andrei Agrachev, Davide Barilari, and Ugo Boscain, A comprehensive introduction to sub-riemannian geometry, 2019, p. 634.

[ALDS19] Andrei A. Ardentov, Enrico Le Donne, and Yuri L. Sachkov, SubFinsler geodesics on the Cartan group, Regul. Chaotic Dyn. 24 (2019), no. 1, 36-60. MR 3908389 
[AS04] Andrei A. Agrachev and Yuri L. Sachkov, Control theory from the geometric viewpoint, Encyclopaedia of Mathematical Sciences, vol. 87, Springer-Verlag, Berlin, 2004, Control Theory and Optimization, II. MR 2062547

[AS15] A. A. Ardentov and Yu. L. Sachkov, Cut time in sub-Riemannian problem on Engel group, ESAIM Control Optim. Calc. Var. 21 (2015), no. 4, 958-988. MR 3395751

[BBI01] Dmitri Burago, Yuri Burago, and Sergei Ivanov, A course in metric geometry, Graduate Studies in Mathematics, vol. 33, American Mathematical Society, Providence, RI, 2001. MR 1835418

[BBLDS17] Davide Barilari, Ugo Boscain, Enrico Le Donne, and Mario Sigalotti, Sub-Finsler structures from the time-optimal control viewpoint for some nilpotent distributions, J. Dyn. Control Syst. 23 (2017), no. 3, 547-575. MR 3657277

[BC18] Z. M. Balogh and A. Calogero, Infinite geodesics of subFinsler distances in the Heisenberg groups, ArXiv e-prints (2018), arXiv:1807.10369.

$\left[\mathrm{BCJ}^{+} 18\right] \quad$ D. Barilari, Y. Chitour, F. Jean, D. Prandi, and M. Sigalotti, On the regularity of abnormal minimizers for rank 2 sub-Riemannian structures, ArXiv e-prints (2018).

[BdSFPR18] André Belotto da Silva, Alessio Figalli, Adam Parusiński, and Ludovic Rifford, Strong Sard Conjecture and regularity of singular minimizing geodesics for analytic sub-Riemannian structures in dimension 3, ArXiv e-prints (2018).

[BFS18] Zoltán M. Balogh, Katrin Fässler, and Hernando Sobrino, Isometric embeddings into Heisenberg groups, Geom. Dedicata 195 (2018), 163192. MR 3820500

[HLD16] Eero Hakavuori and Enrico Le Donne, Non-minimality of corners in subriemannian geometry, Invent. Math. 206 (2016), no. 3, 693-704. MR 3573971

[HLD18] _ Blowups and blowdowns of geodesics in Carnot groups, ArXiv e-prints (2018).

[Kis03] Iwao Kishimoto, Geodesics and isometries of Carnot groups, J. Math. Kyoto Univ. 43 (2003), no. 3, 509-522. MR 2028665

[KLD17] Ville Kivioja and Enrico Le Donne, Isometries of nilpotent metric groups, J. Éc. polytech. Math. 4 (2017), 473-482. MR 3646026

[LD15] Enrico Le Donne, A metric characterization of Carnot groups, Proc. Amer. Math. Soc. 143 (2015), no. 2, 845-849. MR 3283670

[LDO16] Enrico Le Donne and Alessandro Ottazzi, Isometries of Carnot groups and sub-Finsler homogeneous manifolds, J. Geom. Anal. 26 (2016), no. 1, 330-345. MR 3441517

[LDR19] Enrico Le Donne and Séverine Rigot, Besicovitch covering property on graded groups and applications to measure differentiation, J. Reine Angew. Math. 750 (2019), 241-297. MR 3943324

[MPV18a] Roberto Monti, Alessandro Pigati, and Davide Vittone, Existence of tangent lines to Carnot-Carathéodory geodesics, Calc. Var. Partial Differential Equations 57 (2018), no. 3, Art. 75, 18. MR 3795207

[MPV18b] , On tangent cones to length minimizers in CarnotCarathéodory spaces, SIAM J. Control Optim. 56 (2018), no. 5, 33513369. MR 3857882 
[Pan89] Pierre Pansu, Métriques de Carnot-Carathéodory et quasiisométries des espaces symétriques de rang un, Ann. of Math. (2) 129 (1989), no. 1, 1-60. MR 979599

[Roc70] R. Tyrrell Rockafellar, Convex analysis, Princeton Mathematical Series, No. 28, Princeton University Press, Princeton, N.J., 1970. MR 0274683

(Hakavuori) SISSA, ViA BonomeA 265, 34136 TRIESTE

E-mail address: eero.hakavuori@sissa.it 